\title{
A Randomized Clinical Trial of a Tailored Lifestyle Inter- vention for Obese, Sedentary, Primary Care Patients
}

\author{
Max Romano, MD, MPH \\ Johns Hopkins University Bloomberg School of Public Health and MedStar Franklin Square Medical Center, Baltimore, Maryland
}

Ann Fam Med 2016;14(4):iii. doi:10.1370/afm.1963.

T The Annals of Family Medicine encourages readers to develop a learning community of those seeking to improve health care and health through enhanced primary care. You can participate by conducting a RADICAL journal club and sharing the results of your discussions in the Annals online discussion for the featured articles. RADICAL is an acronym for Read, Ask, Discuss, Inquire, Collaborate, Act, and Learn. The word radical also indicates the need to engage diverse participants in thinking critically about important issues affecting primary care and then acting on those discussions. ${ }^{1}$

\section{HOW IT WORKS}

In each issue, the Annals selects an article or articles and provides discussion tips and questions. We encourage you to take a RADICAL approach to these materials and to post a summary of your conversation in our online discussion. (Open the article online and click on "TRACK Comments: Submit a response.") You can find discussion questions and more information online at: http://www.AnnFamMed.org/AJC/.

\section{CURRENT SELECTION}

\section{Article for Discussion}

Eaton CB, Hartman S, Perzanowski E, et al. A randomized clinical trial of a tailored lifestyle intervention for obese, sedentary, primary care patients. Ann Fam Med. 2016;14(4):311-319.

\section{Discussion Tips}

This article provides a chance to consider a pragmatic intervention for one of the country's biggest health problems.

\section{Discussion Questions}

- What question is asked by this study and why does it matter?
- Why did the authors choose this lifestyle intervention? How does this intervention differ from other programs for obese, sedentary adults?

- How appropriate is the block-randomized controlled trial design for answering this question?

- To what degree can the findings be accounted for by:

1. How patients were selected, excluded, randomized, and lost to follow-up?

2. How the main outcome variables were measured or self-reported?

3. Confounding (false attribution of causality because 2 variables discovered to be associated actually are associated with a 3rd factor)? How do the authors attempt to control for confounding in their analysis?

4. Chance?

- What are the main study findings?

- How do you interpret the differences in outcomes after $6,12,18$, and 24 months follow-up?

- How applicable is the study protocol to your practice? Are the study participants similar to your patients? What would be needed to make such an intervention practical in your setting?

- How could this study change your practice? Policy? Education? Research?

- If you designed a new lifestyle intervention for obese, sedentary adults in your own practice, how would it be structured and why? What evidence would you have to support its efficacy?

- What are the next steps in interpreting or applying these findings?

- What researchable questions remain?

\section{References}

1. Stange KC, Miller WL, McLellan LA, et al. Annals Journal Club: It's time to get RADICAL. Ann Fam Med. 2006;4(3):196-197. http:// annfammed.org/cgi/content/full/4/3/196. 